\title{
GENETIC AND COMBINATORIAL ALGORITHMS FOR OPTIMAL SIZING AND PLACEMENT OF ACTIVE POWER FILTERS
}

\author{
MARCIN MACIAZŻEK $^{a, *}$, DARIUSZ GRABOWSKI $^{a}$, MARIAN PASKO $^{a}$ \\ ${ }^{a}$ Institute of Electrical Engineering and Computer Science \\ Silesian University of Technology, ul. Akademicka 10, 44-100 Gliwice, Poland \\ e-mail: marcin.maciazek@polsl.pl
}

\begin{abstract}
The paper deals with cost effective compensator placement and sizing. It becomes one of the most important problems in contemporary electrical networks, in which voltage and current waveform distortions increase year-by-year reaching or even exceeding limit values. The suppression of distortions could be carried out by means of three types of compensators, i.e., passive filters, active power filters and hybrid filters. So far, passive filters have been more popular mainly because of economic reasons, but active and hybrid filters have some advantages which should cause their wider application in the near future. Active power filter placement and sizing could be regarded as an optimization problem. A few objective functions have been proposed for this problem. In this paper we compare solutions obtained by means of combinatorial and genetic approaches. The theoretical discussion is followed by examples of active power filter placement and sizing.
\end{abstract}

Keywords: power quality, optimization, active power filters, harmonics, genetic algorithms, combinatorial algorithms.

\section{Introduction}

Although three phase voltages generated by synchronous generators are sinusoidal and symmetrical, voltages and currents further in the power system are very often distorted. This is caused by either power electronic devices and large asymmetrical loads, e.g., arc furnaces, or by a great number of low power nonlinear loads, e.g., switched-mode power supplies, compact fluorescent lamps, etc. The most popular solution to this problem consists in application of passive filters. However, dynamic changes in waveform distortions in a given power system cross-section make their application less effective in comparison with hybrid and active power filters (Akagi, 2006).

Sizing and placement of compensators have been already considered an optimization task for both passive (Pamplona and Souza, 2004; Chang et al., 2006; He et al., 2009; Dehini and Sefiane, 2011; Carpinelli et al., 2013) and active or hybrid filters (Hong and Chang, 1996; Keypour et al., 2004; Ramos et al., 2006; Dehghani and Ziari, 2008; Yan-Song et al., 2010; Ziari and Jalilian, 2010; 2012; Gehrke et al., 2012b; Moradifar and Soleymanpour, 2012). This paper presents a solution of

\footnotetext{
*Corresponding author
}

the problem for parallel active power filters (APFs), which behave as controlled current sources injecting additional currents into the power system nodes. This results, among other things, in reduction of supplying voltage and current distortions.

The optimization procedure should find the allocation of compensators having minimum nominal currents which ensure an acceptable level of waveform distortions (Kennedy et al., 2006; Yan-Song et al., 2010; Gehrke et al., 2012b; Moradifar and Soleymanpour, 2012; Ziari and Jalilian, 2012). In addition to nominal currents, the objective function could be also defined using voltage total harmonic distortion coefficients, telephone interference factors, motor load losses or harmonic transmission line losses as decision-making variables (Hong and Chang, 1996; Keypour et al., 2004; Ramos et al., 2006; Dehghani and Ziari, 2008; Gehrke et al., 2012a; Grabowski et al., 2013). In this case the maximum compensator nominal currents are defined within constraints of the optimization task. Moreover, a few objective functions could be minimized simultaneously using the multi-objective optimization approach (He et al., 2009; Ziari and Jalilian, 2010; Rafiei et al., 2010; Yue et al., 2011). 
The economic cost is another factor which should be taken into account when solving the problem. In many works it has been assumed that minimization of compensator nominal currents automatically brings about cost reduction because the compensator price depends on its nominal current (Kennedy et al., 2006; Rafiei et al., 2010; Ziari and Jalilian, 2010; González-Romera et al., 2012). This is for sure true if only one compensator is installed, but for more complex problems the objective function directly based on economic cost gives a better final solution. Some results for such an approach were presented by He et al. (2009), Yan-Song et al. (2010), Yue et al. (2011) as well as Ziari and Jalilian (2012). However, contrary to the solution presented in this paper, the cost has been there assumed to be a linear function of the compensator size.

Minimization of the economic cost of active power filter application is of great practical importance. The cost is just one of the factors which limits common use of active power filters. As some accidents all over the world have proved, the low power quality could lead to very far-reaching consequences. The increasing number of nonlinear loads, which are the main cause of low power quality, makes research in this field essential.

On the basis of a review of the literature, the current state of the art in the field of optimal APF allocation could be summarized in a few points:

1. APFs are used as single devices in order to improve power quality. Control algorithms of APFs aim at reaching the sinusoidal shape of line currents at the point of installation.

2. As far as the authors are aware, there are no applications of multiple APFs aimed at a simultaneous power quality improvement in the whole power system under consideration. However, some research work in this direction has been already carried out using the optimization approach.

3. The main difference between the solutions proposed so far consists in the definition of the objective function and constraints. However, there is still room for new propositions, especially the ones which convince potential investors that the cost of the solution could be considerably reduced reaching an acceptable level of waveform distortions. An economic point of view should be a crucial factor in the proposed solutions. Unfortunately, it has been very often omitted or taken into account with some simplifying assumptions leading to sub-optimal solutions.

4. Many optimization algorithms, including emergent gradient and non-gradient algorithms, have been applied to solve the optimization problem under consideration. It seems that recent advances in optimization theory have been fully utilized to find the best solution, and this is not the point for which any breakthrough is required.

5. A solution to the problem requires both electric power flow analysis and advanced optimization algorithms. There is no dedicated software which allows realizing both tasks simultaneously. It also limits the number of research projects undertaken in this area.

The main contribution of this paper to the current state of the art in the field consists in the following:

1. The proposition of an objective function which allows reducing compensation cost to an economically reasonable limit-it ensures that investment costs required to keep power quality indices, e.g., the voltage total harmonic distortion coefficient THDV, below some thresholds will not exceed the assumed levels. The proposed objective function could be compared to the quality/price ratio used in economic analysis. Such an approach has not been used so far and in our opinion it could be applied in practice, especially because high APF costs cause that very often investors use less efficient but cheaper passive filters.

2. The development of complete optimization algorithms which enable allocation of APFs in specific power systems including distributed nonlinear loads in accordance with the proposed objective function.

3. Implementation of a universal software system, based on PCFLO, Matlab and Java, which enables simultaneous analysis of the harmonic flow and application of arbitrary optimization methods for allocation of active as well as passive filters.

\section{Active power filters}

Parallel active power filters can be modeled as controlled current sources injecting additional currents into the power system nodes, cf. Fig. 1. The current of such a compensator connected to a bus $w$ can be described by the Fourier series (the phase index has been omitted, the superscript $k$ denotes the compensator current):

$$
i_{w}^{\mathrm{k}}(t)=\sqrt{2} \operatorname{Re} \sum_{h=1}^{H} I_{w h}^{\mathrm{k}} e^{j h \omega_{0} t},
$$

where $H$ is the highest harmonic number, $I_{w h}^{\mathrm{k}}$ is the phasor of the $h$ harmonic,

$$
I_{w h}^{\mathrm{k}}=\left|I_{w h}^{\mathrm{k}}\right| \mathrm{e}^{j \varphi_{w h}^{\mathrm{k}}},
$$




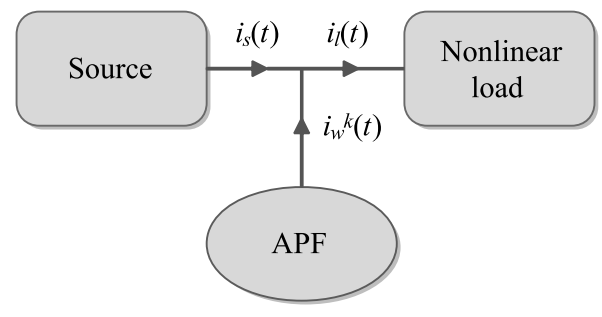

Fig. 1. Compensation idea by means of an APF.

$\left|I_{w h}^{\mathrm{k}}\right|$ is the RMS value of the $h$ harmonic, $\varphi_{w h}^{\mathrm{k}}$ is the phase of the $h$ harmonic.

Active power filters are usually controlled by a classical algorithm (local distortion reduction), i.e., an APF injects currents which cause the line currents at the point of installation to be as close to a sine signal as possible (Maciążek, 2012). In the paper we consider also a modified algorithm (global distortion reduction) in which an APF injects currents which cause the maximum possible suppression of voltage waveform distortions at the whole network under consideration. Such an approach requires information about currents and voltages at all network nodes, and is similar to network-wide harmonic reduction and cooperative control introduced by Kennedy et al. (2006), Gehrke et al. (2012a) and González-Romera et al. (2012).

\section{Objective functions}

An objective function and some constraints must be defined in order to apply the optimization approach to allocation and sizing of compensators. The decision-making variables usually include compensator current phasors as well as indexes of buses to which compensators should be connected. An overview of objective functions for the problem under consideration is given by Grabowski and Walczak (2012). Objective functions used in this paper are defined in the subsequent sections.

3.1. Cost effective objective function. The most popular objective function for the problem of compensator allocation is usually defined as the sum of RMS values of all compensator currents (which can take continuous or discrete values):

$$
\underset{\left\{\operatorname{Re}\left(I_{\boldsymbol{T}[w] h}^{\mathrm{k}}\right), \operatorname{Im}\left(I_{\boldsymbol{T}[w] h}^{\mathrm{k}}\right), \boldsymbol{T}[w]\right\}}{\operatorname{minimize}} \quad f_{1}=\sum_{w=1}^{W}\left|I_{\boldsymbol{T}[w]}^{\mathrm{k}}\right|,
$$

where

$\operatorname{Re}\left(I_{\boldsymbol{T}[w] h}^{\mathrm{k}}\right), \operatorname{Im}\left(I_{\boldsymbol{T}[w] h}^{\mathrm{k}}\right)$ are real and imaginary parts of the $h$ harmonic of the current phasor for the compensator number $w$ placed in a bus defined by $\boldsymbol{T}[w]$; if APFs are used also for power factor correction then $h=1,2,3, \ldots, H$, otherwise $h=$ $2,3, \ldots, H$;

$W$ is the number of compensators to be installed in the system;

$N$ is the total number of buses in the analysed system;

$S$ is the set of indexes of buses to which compensators could be attached in the analysed system, $S=$ $\left\{s_{1}, s_{2}, \ldots, s_{K}\right\}, K \leq N$;

$\boldsymbol{T}$ is the vector containing indexes of buses to which compensators should be attached, $\boldsymbol{T}[w] \in S, w=$ $1,2, \ldots, W, \operatorname{dim}[\boldsymbol{T}]=W$, for example, if $W=2$ and $\boldsymbol{T}=[14]$, then there are 2 compensators located in buses 1 and 4;

$\left|I_{T[w]}^{\mathrm{k}}\right|$ is the RMS of the current for a compensator number $w$ placed in the bus defined by $\boldsymbol{T}[w]$ (if the APF is not used for power factor correction, the index $h$ starts from 2):

$$
\begin{aligned}
& \left|I_{\boldsymbol{T}[w]}^{\mathrm{k}}\right| \\
& =\sqrt{\sum_{h=1}^{H}\left(\left(\operatorname{Re}\left(I_{\boldsymbol{T}[w] h}^{\mathrm{k}}\right)\right)^{2}+\left(\operatorname{Im}\left(I_{\boldsymbol{T}[w] h}^{\mathrm{k}}\right)\right)^{2}\right)} .
\end{aligned}
$$

The optimization problem defined by Eqn. (3) could be solved taking into account some or all constraints listed below:

1. RMS values of compensator currents must be lower than the maximum acceptable value $\left|I^{\mathrm{k}}\right|_{\max }$, i.e., for $w=1,2, \ldots, W$, the following relation must be fulfilled:

$$
\left|I_{T[w]}^{\mathrm{k}}\right| \leq\left|I^{\mathrm{k}}\right|_{\max }
$$

2. RMS values of voltage harmonics in all buses must be lower than the maximum acceptable values $\left|V_{h}\right|_{\max }$, i.e., for $b=1,2, \ldots, N$ and $h=$ $2,3, \ldots, H$, the following relation must be fulfilled:

$$
\left|V_{b h}\right| \leq\left|V_{h}\right|_{\max }
$$

3. Values of voltage THD coefficients in all buses must be lower than the maximum acceptable value $\mathrm{THDV}_{\max }$, i.e., for $b=1,2, \ldots, N$, the following relation must be fulfilled:

$$
\mathrm{THDV}_{b} \leq \mathrm{THDV}_{\text {max }}
$$

4. RMS values of compensator $h$ harmonic currents must be lower than the maximum values $\left|I_{h}^{\mathrm{k}}\right|_{\max }$ given by the compensator manufacturer, i.e., for $w=1,2, \ldots, W$, and $h=1,2, \ldots, H$ the following relation must be fulfilled:

$$
\left|I_{\boldsymbol{T}[w] h}^{\mathrm{k}}\right| \leq\left|I_{h}^{\mathrm{k}}\right|_{\max } .
$$




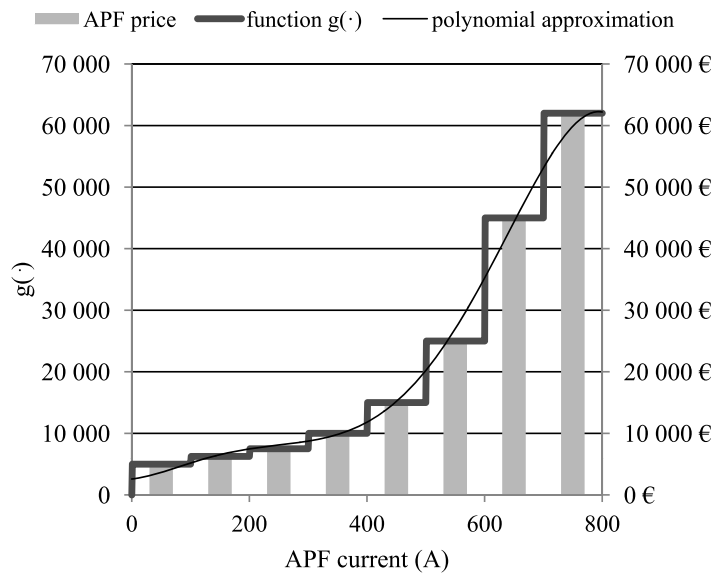

Fig. 2. Sample function describing the relation between APF price and size.

The economic criterion could be exactly applied by including a nonlinear function, reflecting the relation between the compensator nominal current and its price as well as other costs if required (see Fig. 2), into the objective function defined by Eqn. (3):

$$
\underset{\left\{\operatorname{Re}\left(I_{\boldsymbol{T}[w] h}^{\mathrm{k}}\right), \operatorname{Im}\left(I_{\boldsymbol{T}[w] h}^{\mathrm{k}}\right), \boldsymbol{T}[w]\right\}}{\operatorname{minimize}} \quad f_{2}=\sum_{w=1}^{W} g\left(\left|I_{\boldsymbol{T}[w]}^{\mathrm{k}}\right|\right) .
$$

Because the nominal currents belong to a discrete set, the function $g(\cdot)$ is not continuous and its step shape reflects the pricing policy as well as the available product range. As a result, gradient optimization methods cannot be directly used. A solution to the problem consists in either approximation of the function $g(\cdot)$ or application of non-gradient algorithms (Mouatasim et al., 2011).

3.2. Combinatorial objective functions. If the combinatorial approach has been selected, then in the simplest case the optimization is performed separately for all possible combinations of $W$ compensator allocations, i.e., for all possible vectors $\boldsymbol{T}$. Thus, in this case the vector $\boldsymbol{T}$ is no longer included in the set of decision-making variables:

$$
\underset{\left\{\operatorname{Re}\left(I_{\boldsymbol{T}[w] h}^{\mathrm{k}}\right), \operatorname{Im}\left(I_{\boldsymbol{T}[w] h}^{\mathrm{k}}\right)\right\}}{\operatorname{minimize}} \quad f_{2}=\sum_{w=1}^{W} g\left(\left|I_{\boldsymbol{T}[w]}^{\mathrm{k}}\right|\right) .
$$

Indexes of the buses to which compensators should be attached are determined after solving the optimization problem (10) for all $M$ combinations-see Section 4.1 The number $M$ of $W$ combinations from a given set $S$ of buses, such that the order of selection does not matter, is equal to the binomial coefficient, i.e., $M=\left(\begin{array}{c}|S| \\ W\end{array}\right)$, $W \leq|S|$, where $|S|$ denotes the cardinality of the set $S$. Therefore, the optimization problem 10 is solved
$M$ times, each time giving an optimum value $F_{2}(m)$, $m=1,2, \ldots, M$. Next, in the successive step of the algorithm, the best solution is chosen.

The objective functions $f_{1}$ and $f_{2}$ assume that the compensator plays the role of a current source, which can be freely adjustable within a given range in order to obtain the required distortion level in the whole power system under consideration. As mentioned in Section 2, classical APF control algorithms allow rather decreasing current distortions (represented by the current total harmonic distortion coefficient-THDI) at the point of APF connection. It results in an almost-a sinusoidal shape of the line current in the bus under consideration-local approach. The optimization problem for this case consists of a set of $W$ optimization local subproblems ( $w=$ $1,2, \ldots, W)$ solved simultaneously:

$$
\underset{\left\{\operatorname{Re}\left(I_{\boldsymbol{T}[w] h}^{\mathrm{k}}\right), \operatorname{Im}\left(I_{\boldsymbol{T}[w] h}^{\mathrm{k}}\right)\right\}}{\operatorname{minimize}} \quad f_{3}=\mathrm{THDI}_{\boldsymbol{T}[w]} .
$$

This procedure is repeated for successive combinations of compensator allocations, i.e., for $m=1,2, \ldots, M$.

\section{Numerical algorithms}

A numerical algorithm used to solve the optimization problem is another important factor, besides the objective function definition, influencing the results. So far, the GBDT algorithm (Hong and Chang, 1996), combinatorial algorithms (Yamamoto et al., 1999; Dzieciątko, 2001; Pamplona and Souza, 2004; Maciążek et al., 2013), the TABU algorithm (Yan-Song et al., 2010), modified adaptive or discrete particle swarm algorithms (He et al., 2009; Ziari and Jalilian, 2010; 2012; Moradifar and Soleymanpour, 2012) and genetic algorithms (Keypour et al., 2004; Ramos et al., 2006; Dehghani and Ziari, 2008; Yue et al., 2011; Grabowski and Maciążek, 2012) have been applied to solve the problem of compensator allocation and sizing. In this paper we use a combinatorial algorithm, which can be preferred to solve lower-size problems, and a genetic algorithm. The results obtained for both the approaches are compared. The factor graph based genetic algorithm could be used in the future to encode the underlying dependencies between variables of the problem for more complex systems (Helmi et al., 2014).

4.1. Combinatorial algorithm. In the case of the combinatorial approach the final APF allocation is chosen after solving the problem (10) for all combinations. As a result, an additional optimization problem must be solved to determine indexes of the buses with compensators:

$$
\text { arg } \min _{m \in\{1,2, \ldots, M\}} F_{2}(m),
$$

where $F_{2}(m)$ is solution to the problem 10$)$ for the $m$-th combination. 
The solutions obtained using the objective function defined by 11 could be compared by introducing an auxiliary function:

$$
F_{3}(m)=\sum_{w=1}^{W} g\left(\left|I_{\boldsymbol{T}[w]}^{\mathrm{k}}\right|\right) .
$$

The decision in which buses the compensators should be attached is made in a way similar to the formula (12) using the function $F_{3}(m)$ instead of $F_{2}(m)$.

In order to take into account the economic criterion and the constraint on the THDV value, the choice of the solution obtained with the help of (11) could be made by finding the minimum positive value of the function

$$
F_{4}(m)=\frac{\max _{b} \operatorname{THDV}_{b}^{[m+1]}-\max _{b} \operatorname{THDV}_{b}^{[m]}}{\sum_{w=1}^{W} g\left(\left|I_{\boldsymbol{T}[w]}^{\mathrm{k}}\right|[m+1]\right)-\sum_{w=1}^{W} g\left(\left|I_{\boldsymbol{T}[w]}^{\mathrm{k}}\right|[m]\right)},
$$

where $m$ is the index pointing at successive solutions obtained using (11) and sorted in ascending order in relation to the maximum value of $\mathrm{THDV}_{b}, \quad b=$ $1,2, \ldots, N$, and $\left|I_{\boldsymbol{T}[w]}^{\mathrm{k}}\right|^{[m]}$ is the RMS value of the current for a compensator number $w$ placed in a bus defined by $\boldsymbol{T}[w]$ in the case of the solution number $m$.

The objective function (14), which enables selection of an optimal solution from the economical point of view, is a kind of compromise between power quality improvement and financial cost required to achieve this improvement. In accordance with research carried out in some academic centers, the standards defining maximum allowable higher harmonic content are sometimes too restrictive (Fuchs et al., 2004). Moreover, different levels of THDV are used across the world, e.g., $5 \%$ and $8 \%$. In general, the possibility of using such a compromise depends always on the specific system and the sensitivity of the loads to the supplying voltage distortion. Nevertheless, it can lead to significant reduction in investment cost, which enables sufficient power quality improvement.

The block diagram of the combinatorial algorithm is presented in Fig. 3 It covers all three cases considered in this section, i.e., application of auxiliary functions $F_{2}(m)$, $F_{3}(m)$ and $F_{4}(m)$. In the first step a set of buses to which APFs can be connected is determined. It has been assumed that it includes buses with nonlinear loads. In the second step of the algorithm the number of APFs is set and the optimization process for successive combination is performed. The last step consists in analysis of results. Finally, the optimum solution for the assumed auxiliary function is chosen.

4.2. Genetic algorithm. Genetic algorithms are well known for their ability to find global optimum

\section{START}

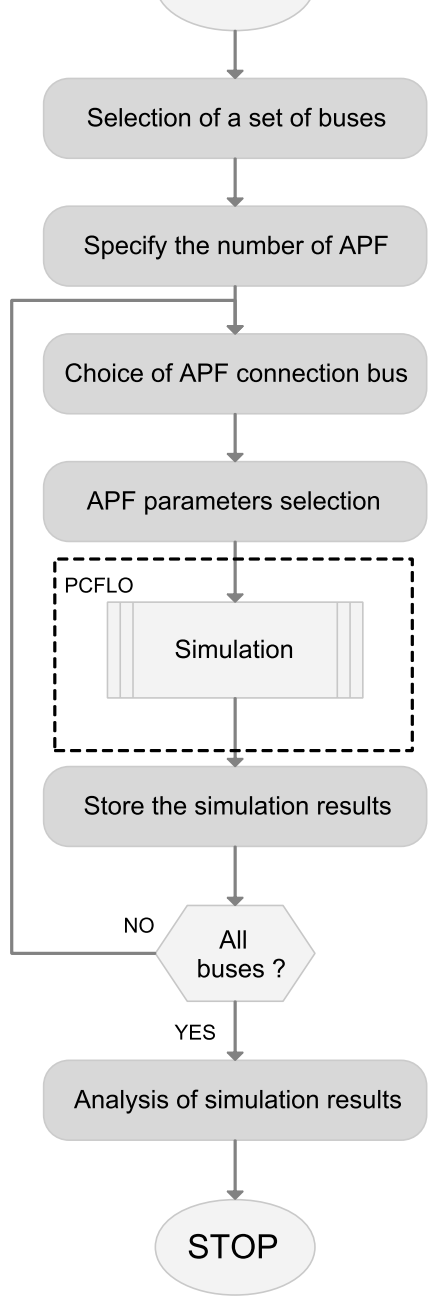

Fig. 3. Block diagram of the combinatorial algorithm.

of complex nonlinear functions using crossover and mutation operators applied to chromosomes coding the solution. The basic parameters of genetic algorithms include mutation and crossover probabilities, the method of data representation, population size, the crossover type, selection rule. The block diagram of a sample chromosome structure coding the decision-making variables for the problem under consideration is presented in Fig. 4 ( $A$ and $B$ denote respectively real and imaginary parts of APF current harmonics in successive buses, i.e., $A_{w_{1}, 2}^{\mathrm{k}}=\operatorname{Re}\left(I_{w_{1} 2}^{\mathrm{k}}\right)$, $B_{w_{1}, 2}^{\mathrm{k}}=\operatorname{Im}\left(I_{w_{1} 2}^{\mathrm{k}}\right)$, etc. $)$

Compensator allocation and sizing is a mixed integer optimization problem because some decision-making variables, namely, those coding to which buses compensators should be connected, are integer-valued. As a result, the genetic algorithm used to solve the problem differs from the basic well-known genetic algorithm by 


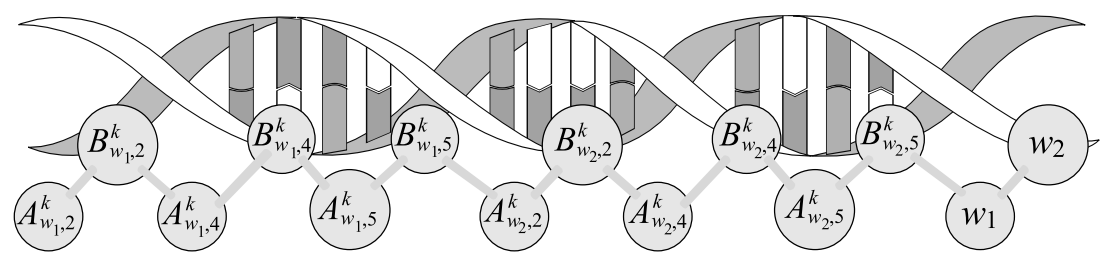

Fig. 4. Chromosome structure for $W=2, H=5$ and no zero sequence components.

two points (Deep and Mohan, 2009):

(i) Some special mutation and crossover operators must be used. They ensure that integer-valued variables in successive generations take only integer values.

(ii) The selection process is based on the binary tournament selection function instead of the roulette-wheel selection rule used for the basic genetic algorithm.

\section{Test system}

There are some test systems which could be used for harmonic analysis, proposed by the IEEE group called the Task Force on Harmonics Modeling and Simulation (Bonner et al., 1996; Abu-Hashim et al., 1999):

- 14-bus test system,

- 13-bus test system I,

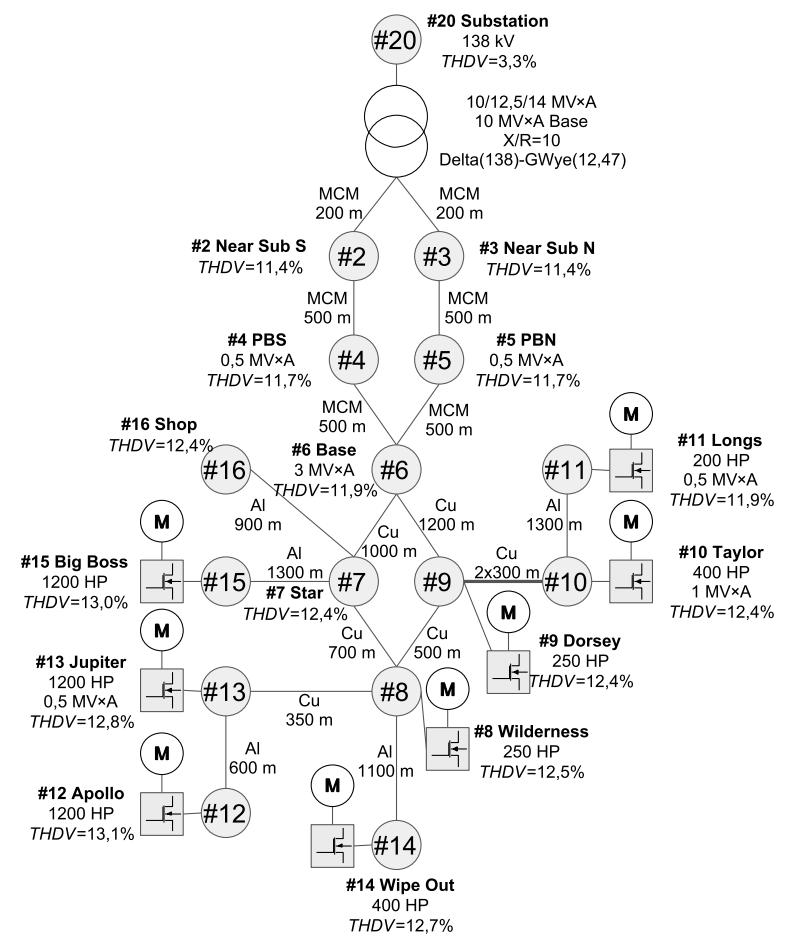

Fig. 5. Test system.
- 13-bus test system II.

Moreover, many other models which enable power flow analysis in power systems have been developed, e.g., the IEEE 30-bus system, IEEE 57-bus system and even the IEEE 300-bus system (Christie, 1993). Unfortunately, their application in harmonic analysis studies is not straightforward because only parameters for the fundamental frequency are directly given and they do not include any nonlinear loads. This is why we decided to use a 17-bus system described by Grady (2010) as a test power system (Fig. 5). It includes 8 buses supplying DC motors driven by six-pulse line-commutated adjustable speed drives, which are the main source of harmonics - the original values of THDV coefficients calculated with the help of the PCFLO software are presented in Fig. 5. The analysis of the system in PCFLO is performed for steady state under some assumptions which are usually made in the case of the harmonic analysis of power systems, i.e,

- for each harmonic, any nonlinear load can be represented by a current source,

- the impedance matrix describing the system can be determined separately for each frequency.

\section{Software}

Harmonic flow analysis for the problem under consideration has been made with the help of PCFLO software (Grady, 2010). Unfortunately, it does not include any options for automatic analysis performed for a few sets of parameters. The only way to overcome this difficulty is to run it in the batch mode, i.e., using the command line and reading all the parameters and data necessary for the simulation from text files. The description of the input-output files is given in Table 1.

The batch mode allows running PCFLO by an external application which has access to the operating system command line. This feature, together with PCFLO input file modification capabilities by external applications, enables to freely control both the simulation parameters and the analysed power system parameters. Matlab has been chosen as the upper level application because of the availability of advanced optimization and result analysis toolboxes. However, this cooperation of 


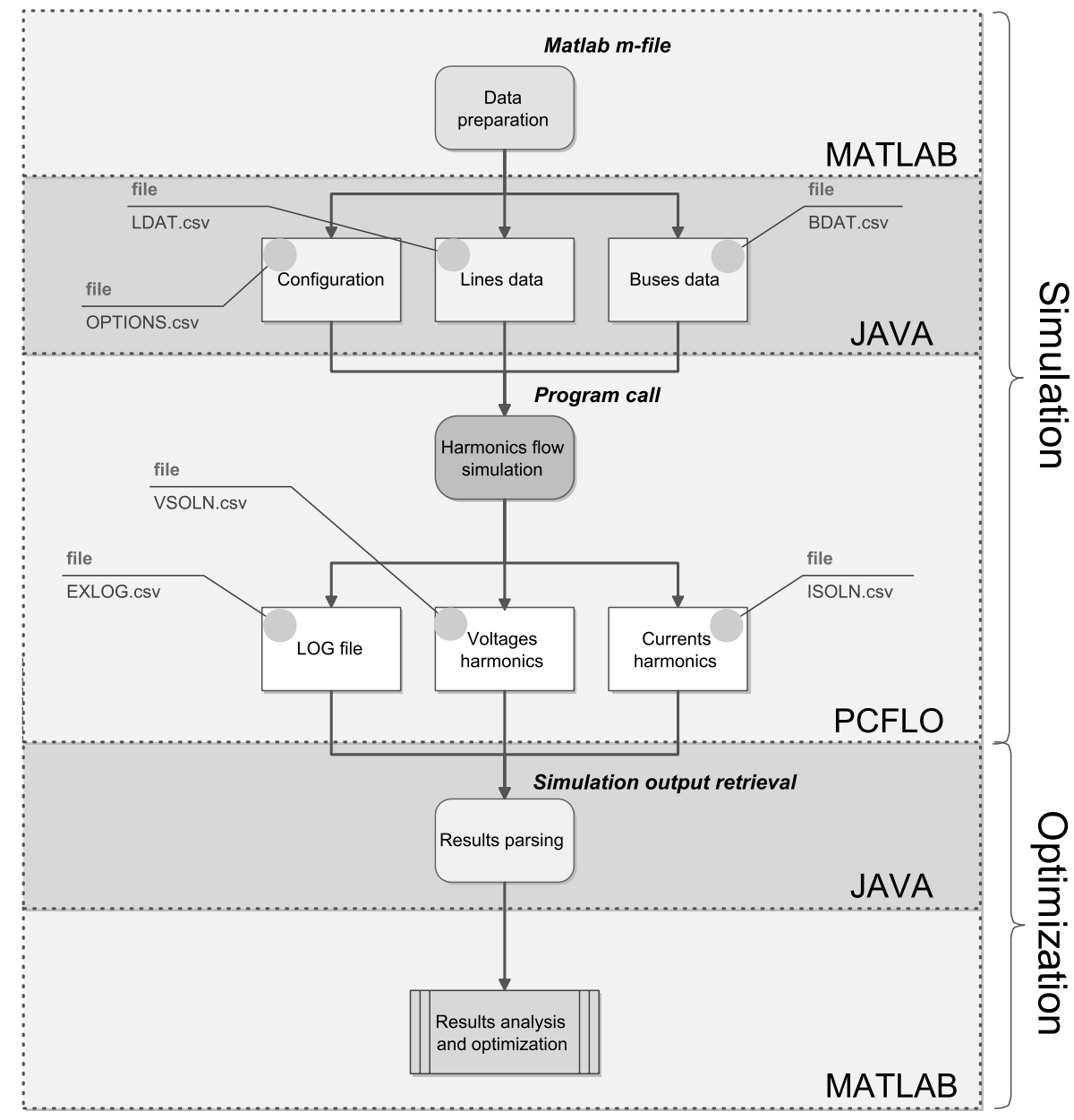

Fig. 6. Task division and exchange of data between Matlab and PCFLO.

Matlab and PCFLO required writing a library of functions which enable easy generation and reading of input and output PCFLO text files in Matlab (Lewandowski et al., 2011). This library was implemented in Java due to the lack of advanced text processing functions in Matlab. Moreover, external libraries written in Java could be easily linked with Matlab. The library includes 17 classes which enable direct access to input and output data files.

Table 1. Files used for information exchange in PCFLO.

\begin{tabular}{|l|l|l|}
\hline \multicolumn{2}{|c|}{ File name } & \multicolumn{1}{c|}{ Description } \\
\hline \hline \multirow{2}{*}{} & BDAT.csv & Bus data \\
\cline { 2 - 3 } & LDAT.csv & Line and transformer data \\
\cline { 2 - 3 } & OPTIONS.csv & Analysis options \\
\cline { 2 - 3 } & SPECTRA.csv & $\begin{array}{l}\text { User-defined models of current } \\
\text { sources with a given spectrum }\end{array}$ \\
\hline \multirow{2}{*}{} & EXLOG.csv & Simulation LOG file \\
\cline { 2 - 3 } & ISOLN.csv & $\begin{array}{l}\text { Current and voltage spectra } \\
\text { for all circuit lines and buses }\end{array}$ \\
\hline \multirow{2}{*}{} & VSOLN.csv & $\begin{array}{l}\text { THD coefficients } \\
\text { for all currents and voltages }\end{array}$ \\
\cline { 2 - 3 } & THDV.csv & \\
\hline
\end{tabular}

A general architecture of the system is shown in Fig. 6,

The cooperation of PCFLO with Matlab increases significantly its capabilities when analysing the harmonic flow in power systems. First of all, it enables optimal allocation of compensators with the help of optimization algorithms implemented in Matlab.

\section{Simulation results}

This section includes solutions of the APF allocation and sizing problem for the test system (Fig. 5). It has been assumed that APFs can be allocated in buses with numbers belonging to a set $S=\left\{s_{1}, s_{2}, \ldots, s_{14}\right\}=$ $\{1,2,4,6,7,8,9,10,11,12,13,14,15,16\}$.

Example 1. (Combinatorial algorithm) Application of the combinatorial algorithm presented in Fig. 3 had given a set of solutions for the test system which subsequently were sorted in order to suit the objective function (14). Analysis and comparison of results from the economic point of view is easier assuming a reference point. Further, the cost of full compensation using 
the relation shown in Fig. 2 was used as the reference point. Full compensation means both compensation of higher harmonics and reactive power compensation for the fundamental frequency, i.e., power factor correction. It is the most expensive solution which requires allocation of an APF in each bus with a nonlinear load-the relative cost of the compensation for this case is equal to $100 \%$. Analysis of results was made with the help of a dedicated script (m-file) in Matlab. The relative cost of the final solution, for which the maximum THDV in the test system is less than $6.77 \%$, is equal to $33.0 \%$, see Fig. 7 . In accordance with this solution, APFs should be installed in buses: $8\left(\left|I_{8}^{k}\right|=125 A\right), 9\left(\left|I_{9}^{k}\right|=125 A\right), 10\left(\left|I_{10}^{k}\right|=\right.$ 205A), $14\left(\left|I_{14}^{k}\right|=205 A\right)$ and $15\left(\left|I_{15}^{k}\right|=645 A\right)$.

Power factor correction is a very important problem in power systems for which passive filters can be used instead of APFs - the APF function in the power system depends on the control algorithm (Maciążek, 2012). Of course, it decreases the economic cost because the ratings of the APFs are lower if the power factor is corrected by passive filters. Application of the combinatorial algorithm and performing an analysis similar to the previous case leads to results presented in Fig. 8 The relative cost of the final solution, for which the maximum THDV in the test system is less than $7.03 \%$, is equal to $12.5 \%$. The cost of full higher harmonic compensation is equal to $26.2 \%$. Accordingly, the cost for both cases, i.e., economic and full compensation, is about 3 times lower if the power factor correction is made by passive filters. In accordance with this solution, APFs should be installed in buses: 8 $\left(\left|I_{8}^{k}\right|=65 A\right), 9\left(\left|I_{9}^{k}\right|=65 A\right), 10\left(\left|I_{10}^{k}\right|=95 A\right), 13$ $\left(\left|I_{13}^{k}\right|=295 A\right)$ and $14\left(\left|I_{14}^{k}\right|=95 A\right)$.

Application of the combinatorial approach represented by (10) and (12) allows finding an even less expensive solution-the relative cost is equal to $8.0 \%$, and there are only 2 APFs located in buses 12 and 16. The maximum THDV is less than $5.30 \%$. However, in this case the modified control algorithm of APFs,

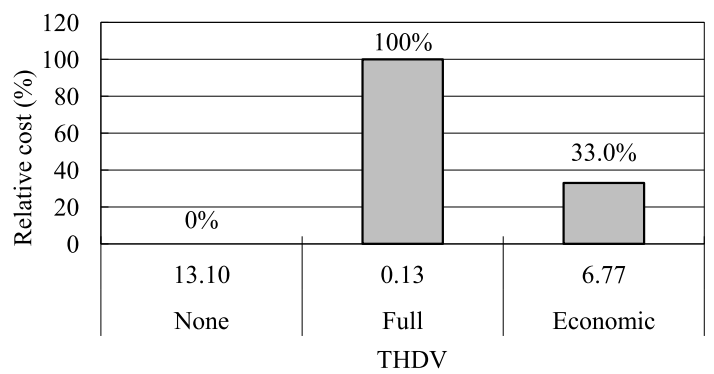

Fig. 7. Comparison of relative costs and maximum THDV coefficients before compensation as well as after full and economic compensation by means of the combinatorial algorithm: APFs used also for power factor correction.

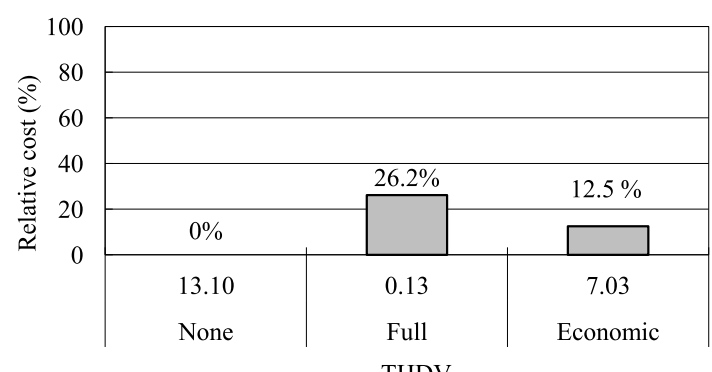

Fig. 8. Comparison of relative costs and maximum THDV coefficients before compensation as well as after full and economic compensation by means of the combinatorial algorithm: power factor correction using passive filters.

which is more complex, must be used-see Section 2

Example 2. (Genetic algorithm) The genetic algorithm described in Section 4.2 has been applied to solve the optimization problem (9). Allocation of compensators in successive generations of the algorithm for $W=2$ is presented in Fig. 9 The allocation is determined with the help of an auxiliary vector $\boldsymbol{T}_{B}$ which defines relationship between elements of the vector $\boldsymbol{T}$ and the set $S$, namely, $\boldsymbol{T}[w]=S_{\boldsymbol{T}_{B}[w]}$. On the basis of Fig. 9, the final values of the auxiliary vector are $\boldsymbol{T}_{B}[1]=6$ and $\boldsymbol{T}_{B}[2]=13$. Thus, keeping in mind that $S=\left\{s_{1}, s_{2}, \ldots, s_{14}\right\}=$ $\{1,2,4,6,7,8,9,10,11,12,13,14,15,16\}$, the APFs are allocated in buses $\boldsymbol{T}[1]=s_{\boldsymbol{T}_{B}[1]}=s_{6}=8$ and $\boldsymbol{T}[2]=$ $s_{\boldsymbol{T}_{B}[2]}=s_{13}=15$.

The relative cost of the final solution is equal to 9.1\%, see Fig. 10 In accordance with this solution, rated currents of APFs are as follows: $\left|I_{8}^{k}\right|=400 A$ and $\left|I_{15}^{k}\right|=$

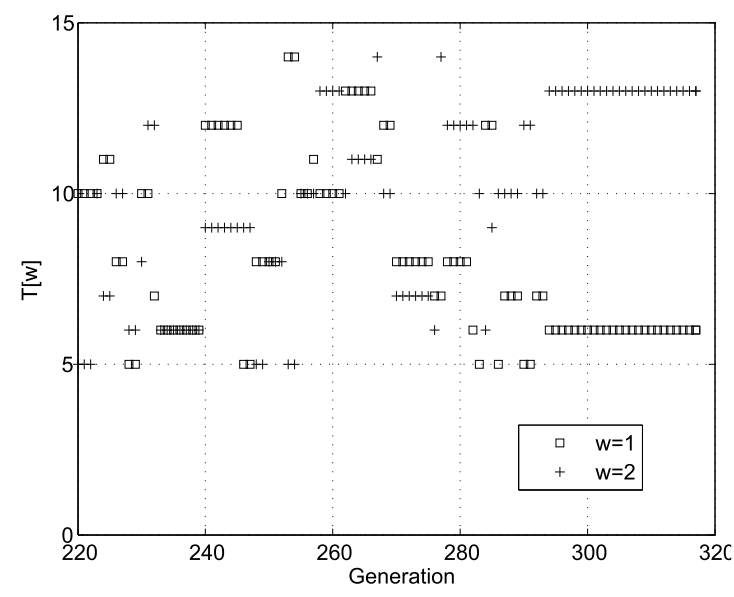

Fig. 9. Allocation of compensators in successive generations of the genetic algorithm for $W=2$. 


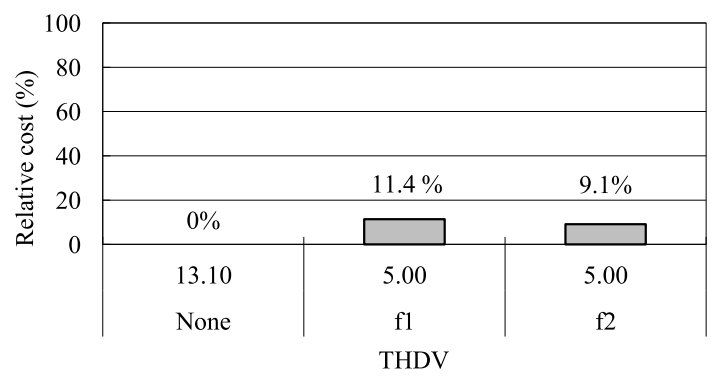

Fig. 10. Comparison of relative costs and maximum THDV coefficients before compensation as well as after full and economic compensation by means of the genetic algorithm: power factor correction using passive filters.

$397 \mathrm{~A}$. If the objective function $f_{1}$ is used instead of $f_{2}$, then the relative cost of the final solution is larger-it is equal to $11.4 \%$ and requires $3 \mathrm{APFs}$ placed in buses 7, 10 and 13 , which have rated currents $\left|I_{7}^{k}\right|=352 \mathrm{~A}$, $\left|I_{10}^{k}\right|=283 A$ and $\left|I_{13}^{k}\right|=259 A$, respectively.

Simulation experiments confirmed that minimization of the compensator currents (objective function $f_{1}$ ) does not necessary lead to the minimum costs as is usually claimed. Including information about the cost directly in the objective function is necessary if more than one compensator is allocated and the relation between the compensator price and the rated current is nonlinear. Application of such an approach (the objective function $f_{2}$ ) results in a significant cost reduction.

In general, the objective functions $f_{1}$ and $f_{2}$ lead to solutions with higher APF ratings compared with those obtained for the function $f_{3}$. The reason is different control algorithms used for both cases-one enables simultaneous minimization of voltage distortions at local and remote buses and requires information about voltages at all buses, while the other is limited only to local distortion minimization. However, the objective function $f_{3}$ usually gives more APFs and higher costs to reach THDV values less than the levels imposed by standards (e.g., IEEE, 1992). A simpler control algorithm, which requires only locally measured data, is an advantage of this approach.

\section{Conclusions}

The analysis of the harmonic flow in a power system presented in the paper shows that the method of APF allocation influences both the compensation efficiency and the financial cost. From the economic point of view, the best solution for a given THDV level has been obtained by means of the genetic algorithm. If APFs are used not only for higher harmonic compensation but also for power factor corrections, the total cost is about three times greater. It must be stressed that implementation of the best solution requires application of the modified APF control algorithm, which needs information about current and voltage distortions in all buses of the system, and so it is much more complex than the classical APF control algorithm based only on current distortions in the bus of installation.

\section{Acknowledgment}

This work was supported by the Polish Ministry of Science and Higher Education under the project no. N N510 257338.

\section{References}

Abu-Hashim, R., Burch, R., Chang, G., Grady, M., Gunther, E., Halpin, M., Harziadonin, C., Liu, Y., Marz, M., Ortmeyer, T., Rajagopalan, V., Ranade, S., Ribeiro, P., Sim, T. and Xu, W. (1999). Test systems for harmonics modeling and simulation, IEEE Transactions on Power Delivery 14(2): 579-587.

Akagi, H. (2006). Modern active filters and traditional passive filters, Bulletin of the Polish Academy of Sciences: Technical Sciences 54(3): 255-269.

Bonner, A., Grebe, T., Gunther, E., Hopkins, L., Man, M., Mahseredjian, J., Miller, N., Ortmeyer, T., Rajagopalan, V., Ranade, S., Ribeiro, P., Spherling, B., Sims, T. and Xu, W. (1996). Modeling and simulation of the propagation of harmonics in electric power networks, II: Sample systems and examples, IEEE Transactions on Power Delivery 11(1): 466-474.

Carpinelli, G., Ferruzzi, G. and Russo, A. (2013). Trade-off analysis to solve a probabilistic multi-objective problem for passive filtering system planning, International Journal of Emerging Electric Power Systems 14(3): 275-284.

Chang, G., Chu, S.-Y. and Wang, H. (2006). A new method of passive harmonic filter planning for controlling voltage distortion in a power system, IEEE Transactions on Power Delivery 21(1): 305-312.

Christie, R. (1993). Power systems test case archive, www. ee.washington. edu/research/pstca/.

Dehghani, N. and Ziari, I. (2008). Optimal allocation of APLCs using genetic algorithm, Proceedings of 43rd International Universities Power Engineering Conference UPEC, Padova, Italy, pp. 1-4.

Dehini, R. and Sefiane, S. (2011). Power quality and cost improvement by passive power filters synthesis using ant colony algorithm, Journal of Theoretical and Applied Information Technology 23(2): 70-79.

Dzieciątko, M. (2001). Application of Combinatorial Optimization Methods to Solution of Higher Harmonics Compensation Problems, Ph.D. thesis, Warsaw University of Technology, Warsaw.

Fuchs, E., Roesler, D. and Masoum, M. (2004). Are harmonic recommendations according to IEEE and IEC 
too restrictive?, IEEE Transactions on Power Delivery 19(4): 1775-1786.

Gehrke, C.S., Lima, A.M.N. and Oliveira, A.C. (2012a). Cooperative control for active power compensators allocated in distributed networks, Proceedings of the IEEE Energy Conversion Congress and Exposition, Raleigh, NC, USA, pp. 2764-2768.

Gehrke, C.S., Lima, A.M.N. and Oliveira, A.C. (2012b). Evaluating APLCs placement in a power system based on real-time simulation, Proceedings of the IEEE Energy Conversion Congress and Exposition, Raleigh, NC, USA, pp. 2011-2016.

González-Romera, E., Romero-Cadaval, E., Ruíz-Arranz, S. and Milanés-Montero, M. (2012). Overall power quality correction in distribution networks by active power filters, optimization of location and strategy, Przeglqd Elektrotechniczny 88(1A): 51-55.

Grabowski, D. and Maciążek, M. (2012). Cost effective allocation and sizing of active power filters using genetic algorithms, Proceedings of the 12th International Conference on Environment and Electrical Engineering EEEIC, Wroctaw, Poland, pp. 467-472.

Grabowski, D., Maciążek, M. and Pasko, M. (2013). Sizing of active power filters using some optimization strategies, International Journal for Computation and Mathematics in Electrical and Electronic Engineering COMPEL 32(4): 1326-1336.

Grabowski, D. and Walczak, J. (2012). Strategies for optimal allocation and sizing of active power filters, Proceedings of the 11th International Conference on Environment and Electrical Engineering EEEIC, Venice, Italy, pp. 1198-1103.

Grady, W.M. (2010). PCFLO v6 users manual, users . ece. utexas. edu/ grady/.

He, N., Xu, D. and Huang, L. (2009). The application of particle swarm optimization to passive and hybrid active power filter design, IEEE Transactions on Industrial Electronics 56(8): 2841-2851.

Helmi, B.H., Rahmani, A.T. and Pelikan, M. (2014). A factor graph based genetic algorithm, International Journal of Applied Mathematics and Computer Science 24(3): 621-633, DOI: 10.2478/amcs-2014-0045.

Hong, Y.-Y. and Chang, Y.-K. (1996). Determination of locations and sizes for active power line conditioners to reduce harmonics in power systems, IEEE Transactions on Power Delivery 11(3): 1610-1617.

IEEE (1992). IEEE Recommended practices and requirements for harmonic control in electric power systems, Std 5191992.

Deep, K., Singh, K.P., Kansal, M.K. and Mohan, C. (2009). A real coded genetic algorithm for solving integer and mixed integer optimization problems, Applied Mathematics and Computation 212(2): 505-518.

Kennedy, K., Lightbody, G., Yacamini, R., Murray, M. and Kennedy, J. (2006). Online control of an APLC for network-wide harmonic reduction, IEEE Transactions on Power Delivery 21(1): 432-439.
Keypour, R., Seifi, H. and Yazdian-Varjani, A. (2004). Genetic based algorithm for active power filter allocation and sizing, Electric Power Systems Research 71(1): 41-49.

Lewandowski, M., Maciążek, M. and Grabowski, D. (2011). Integration of Matlab and PCFLO for harmonic flow analysis in a power system containing APF, Proceedings of the 34th International Conference on Fundamentals of Electrotechnics and Circuit Theory IC-SPETO, Ustron, Poland, pp. 89-90.

Maciążek, M. (2012). Power theories applications to control active compensators, in G. Benysek and M. Pasko (Eds.), Power Theories for Improved Power Quality, Springer, London, pp. 49-116.

Maciążek, M., Grabowski, D. and Pasko, M. (2013). Active power filters - optimization of sizing and placement, Bulletin of the Polish Academy of Sciences: Technical Sciences 61(4): 847-853.

Moradifar, A. and Soleymanpour, H.R. (2012). A fuzzy based solution for allocation and sizing of multiple active power filters, Journal of Power Electronics 12(5): 830-841.

Mouatasim, A.E., Ellaia, R. and de Cursi, E. (2011). Random perturbation of the projected variable metric method for nonsmooth nonconvex optimization problems with linear constraints, International Journal of Applied Mathematics and Computer Science 21(2): 317-329, DOI: 10.2478/v10006-011-0024-Z.

Pamplona, F.M.P. and Souza, B.A. (2004). Harmonic passive filter planning in radial distribution systems using microgenetic algorithms, Proceedings of the 11th International Conference on Harmonics and Quality of Power, New York, NY, USA, pp. 739-744.

Rafiei, S.M.R., Kordi, M.H., Griva, G. and Yassami, H. (2010). Multi-objective optimization based optimal compensation strategies study for power quality enhancement under distorted voltages, Proceedings of the IEEE International Symposium on Industrial Electronics, Bari, Italy, pp. 3284-3291.

Ramos, D.F.U., Cortes, J., Torres, H., Gallego, L.E., Delgadillo, A. and Buitrago, L. (2006). Implementation of genetic algorithms in ATP for optimal allocation and sizing of active power line conditioners, Proceedings of the IEEE/PES Transmission and Distribution Conference and Exposition, Caracas, Venezuela, pp. 1-5.

Yamamoto, F., Kitamura, A., Fujita, N., Nakanishi, Y. and Nagasawa, M. (1999). A study on optimal locations and sizes of active filters as an additional function of distributed generation systems, Proceedings of the IEEE International Conference on Systems, Man, and Cybernetics SMC, Tokyo, Japan, Vol. 6, pp. 515-520.

Yan-Song, W., Hua, S., Xue-min, L., Jun, L. and Song-bo, G. (2010). Optimal allocation of the active filters based on the TABU algorithm in distribution network, Proceedings of the International Conference on Electrical and Control Engineering ICECE, Wuhan, China, pp. 1418-1421.

Yue, H., Li, G., Zhou, M., Wang, K. and Wang, J. (2011). Multi-objective optimal power filter planning 
in distribution network based on fast nondominated sorting genetic algorithms, Proceedings of the 4th International Conference on Electric Utility Deregulation and Restructuring and Power Technologies, Weihai, China, pp. 234-240.

Ziari, I. and Jalilian, A. (2010). A new approach for allocation and sizing of multiple active power-line conditioners, IEEE Transactions on Power Delivery 25(2): 1026-1035.

Ziari, I. and Jalilian, A. (2012). Optimal placement and sizing of multiple APLCs using a modified discrete PSO, International Journal of Electrical Power and Energy Systems 43(1): 630-639.

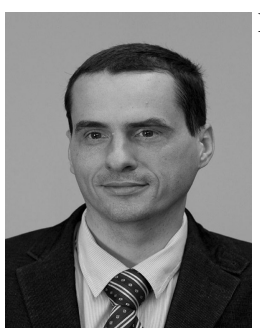

Marcin Maciażek received his M.Sc. (1998) and Ph.D. (2003) degrees in electrical engineering from the Silesian University of Technology. He has taken part in 11 projects supported by the Polish Ministry of Science and Higher Education, being a leader in two of them. He is an author or co-author of over 80 scientific publications, 3 monographs and 3 books. His areas of interest include power quality analysis, methods of power quality improvement by active power filters, and applications of real-time operating systems (RTOs) in embedded systems based on ARM and DSP processors.

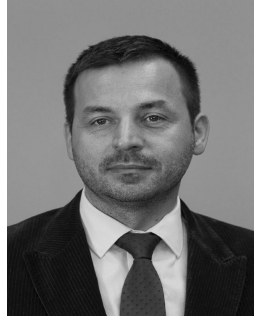

Dariusz Grabowski received his M.Sc. (1993) and Ph.D. (2000) degrees in electrical engineering from the Silesian University of Technology. His Ph.D. thesis was awarded for outstanding scientific achievements by the Rector of the Silesian University of Technology. He was a fellow of the 5th European Community Framework Programme. $\mathrm{He}$ is an author or co-author of over 70 scientific papers, 2 monographs and 2 books. His fields of interest include signal processing, data mining, and application of neural networks and genetic algorithms in electrical and electronic engineering.

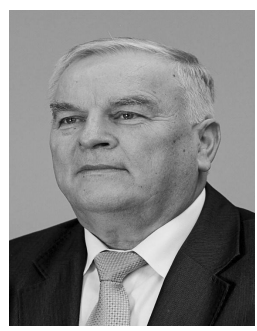

Marian Pasko received his M.Sc. (1971), Ph.D. (1977) and D.Sc. (1994) degrees in electrical engineering from the Silesian University of Technology. He became an associate professor in 1997 and a full professor in 2004. Since 2002 he has been the chapter editor of the Publishing House of the Silesian University of Technology. In 2009 he became the head of the Division of Electrical Machines and Electrical Engineering in Transport. He is an author or a co-author of over 380 scientific papers, 7 monographs and 30 books. His areas of interest include synthesis and analysis of electrical systems, power theory of systems with non-sinusoidal waveforms and sensitivity theory.

Received: 20 May 2014

Revised: 12 September 2014 